\title{
WATERLOO, OÙ LA DÉROUTE DE L'AIGLE EST GLOIRE POSTHUME DU LION
}

\author{
Alain Reyniers ${ }^{1}$
}

Le village de Waterloo est connu dans le monde entier pour une bataille qui ne se déroula pas sur son territoire. C'est, en effet, un peu plus au sud, sur les terres de Braine-l'Alleud, Plancenoit et VieuxGenappe que, le 18 juin 1815, Napoléon affronta pour la dernière fois -et à son désavantage- les Anglo-Hollandais de Wellington et les Prussiens de Blücher ${ }^{2}$. Les combats mirent aux prises plus de 188.000 hommes qui se massacrèrent tout au long de la journée. Quelque 50.000 d'entre-eux allaient y laisser la vie ou y être sérieusement blessés. Le lendemain, au dire d'un agriculteur local, la terre qui avait été violemment pétrie par les chevauchées successives des belligérants ne ressemblait plus qu'à une espèce de pâte gorgée de pluie et de sang. Aujourd'hui, avec bon an mal an un peu plus de 300.000 visiteurs, le site du champ de bataille est, tout comme Bruges et la

1 Chargé de cours invité au Département de communication de l'Université catholique de Louvain.

2 Ce sont les Anglais qui, les premiers, parlèrent de la bataille de Waterloo. Les Français, quant à eux, évoquèrent longtemps la bataille de Mont-Saint-Jean alors que les Prussiens portèrent leur préférence sur la bataille de la Belle-Alliance.

Recherches en communication, $\mathrm{n}^{\circ} 12,(1999)$. 
Grand'Place de Bruxelles, l'un des lieux les plus connus et les plus visités de Belgique.

\section{Un lieu, mémoire d'une bataille}

Incontestablement, une part importante d'un tel succès de masse doit être imputée aux combats titanesques qui ébranlèrent tous les belligérants comme aux conséquences politiques directes de la bataille. Mais cela n'explique pas tout. Car les combats qui se déroulèrent à Waterloo attirent aujourd'hui encore bien plus l'intérêt et les passions des gens que la bataille de l'Yser en 1918 ou celle de Bastogne en 1945. Certes, l'épopée napoléonienne a toujours ses nombreux adeptes de par le monde. Mais la bataille de Waterloo, en tout cas celle qui s'est réellement déroulée sur les terres du Brabant wallon voici près de deux siècles, ne fut guère à l'avantage de l'Empereur, ni sur le plan tactique, ni sur celui des résultats concrets. Alors comment expliquer qu'un échec cuisant soit à ce point le moteur d'une attraction qui ne se dément guère ? On reconnaîtra l'engouement des vainqueurs au lendemain de la tragédie, ou encore le retour des vaincus, cherchant à comprendre, sur les lieux du drame. Mais cela fait bien longtemps que les ennemis de l'époque se sont réconciliés et puis se sont engagés côte à côte dans d'autres combats, pour d'autres enjeux. Le plus curieux, c'est que la défaite même de l'Aigle se soit transformée en victoire posthume. Un échec cuisant et une victoire qui laissent finalement assez peu de gens indifférents. C'est qu'un mythe est attaché à la bataille de Waterloo ; un mythe qui interpelle et tenaille bien plus de monde que les seuls -et nombreuxvisiteurs annuels du site.

Mais, rappelons tout d'abord les faits qui présidèrent aux affrontements de juin $1815^{1}$. Après une première abdication et un exil de

1 Plusieurs centaines d'ouvrages ont été consacrés à la bataille de Waterloo. Le lecteur pourra trouver le point de vue des belligérants, des analyses et un exposé fouillé des diverses phases des combats notamment dans :

- C. von Clausewitz, Campagne de 1815 en France, Traduit de l'allemand par M. Niessel, Paris, IVREA, 1993.

- B. Coppens \& P. Courcelle, Hougoumont. Waterloo 1815. Les Carnets de la Campagne- $N^{\circ} 1$. Bruxelles, Éditions de la Belle Alliance, 1999.

- J. Frings, Dictionnaire de la bataille de Waterloo, Braine-l'Alleud, Les guides $1815,1995$.

- H. HousSAYE, Waterloo 1815, Etrépilly, Christian De Bartillat, 1987. 
quelques mois à l'lle d'Elbe, Napoléon revient en France, pousse sur Paris sans rencontrer grande résistance et arrive aux Tuileries le 20 mars 1815. Cependant, les anciens vainqueurs de l'Aigle ne l'entendent pas ainsi. Le 9 juin 1815, ils signent l'acte final du Congrès de Vienne qui ouvre une nouvelle période d'hostilités avec celui qu'elles considèrent comme un usurpateur. Napoléon, qui a organisé de main de maître la montée en puissance puis la concentration discrète de son armée dans le nord de la France, décide de prendre les Alliés de court en Belgique. Apparemment, la surprise est totale. Les premières escarmouches autour de Thuin, les combats contre les Prussiens à Charleroi, à Gilly puis, le 16 juin à Ligny, tournent à l'avantage des Français. Mais Blücher n'est pas battu et parvient à se replier sur Wavre ; dans cette affaire, il n'est guère inquiété par le Maréchal Grouchy chargé pourtant de le poursuivre. Pendant ce temps, l'aile gauche de l'armée française, commandée par le Maréchal Ney, patine aux Quatre-Bras face aux Hollandais que les Anglais auront tardivement secourus. Le lendemain, Genappe est le théâtre de quelques affrontements sans conséquences entre l'avant-garde de l'Empereur et les Anglo-Hollandais qui font apparemment retraite sur Bruxelles. Dans la soirée, et sous une pluie battante, les belligérants sont face à face à Mont-Saint-Jean et à la Belle-Alliance.

La bataille du 18 juin 1815, proprement dite, met aux prises les Français et les troupes anglo-hollandaises que les Prussiens de Blücher rejoignent en fin d'après-midi. Elle va être marquée par des attaques frontales suicidaires d'infanterie et des charges impétueuses de cavalerie du côté des Français, par l'admirable stoïcité des carrés anglo-alliés face à celles-ci. L'arrivée des Prussiens sur le flanc droit des Français va donner lieu à une résistance acharnée de ces derniers à Plancenoit. Néanmoins, submergées en fin de journée par un ennemi nettement supérieur, brisées par la fatigue et rongées par un indicible

- S. DELloye, Napoléon dans sa dernière campagne. Itinéraire du 12 au 21 juin 1815, Vieux-Genappe, Société Belge d'Etudes Napoléoniennes, 1997.

- J. LogIE, Waterloo, l'évitable défaite, Paris-Gembloux, Duculot, 1984.

- J. LoGie, (coord.), Waterloo 1815. L'Europe face à Napoléon, Bruxelles, Crédit Communal, 1990.

- P. MAES, Juin 1815... Radioscopie d'une campagne, Cahier $\mathrm{n}^{\circ} 12$, Ligny, Association Belge Napoléonienne Edition, 1995.

- H. T. SibORNE, (éd), Waterloo Letters, London, Greenhill Books, 1993.

- W. SIBORNE, History of the Waterloo Campaign, London, Greenhill Books., 1995

- A. UfFINDEL \& M. CORUM, On the Fields of Glory. The Battlefields of the 1815 Campaign, London, Greenhill Books, 1996. 
sentiment de trahison, les troupes de Napoléon cèdent le terrain et sombrent dans la déroute. La débâcle sera toutefois évitée par la résistance héroïque de quelques bataillons appartenant à la Garde impériale. En fin de journée, le spectacle offert par le champ de bataille est terrifiant: plusieurs dizaines de milliers de morts et de blessés jonchent le sol. Les vainqueurs n'avaient pas prévu cela et mettront du temps pour organiser les secours comme le nettoyage du site. Il faudra plusieurs jours pour relever les combattants qui pouvaient encore l'être et pour enterrer les très nombreux cadavres. Plusieurs centaines de corps furent incinérés ; les autres allaient être inhumés dans de vastes fosses communes, parfois même simplement jetés dans les dépressions du terrain et sommairement recouverts.

La victoire des uns et la défaite des autres va faire l'objet de nombreux commentaires. Napoléon chargera ses subordonnés ; les critiques lui reprocheront de ne pas avoir évalué la détermination des alliés à son encontre et de ne pas avoir développé sur le terrain une tactique plus manœuvrière. Son apathie sera soulignée, tout comme l'incurie de son état-major: "on m'a fait manœuvrer comme des patates", dira plus tard le colonel Marbot dont les hussards furent chargés d'observer puis de contenir les premiers Prussiens. Le mauvais temps, une succession de retards malencontreux, des lacunes dans la connaissance du terrain, des ordres mal compris ou mal transmis, tout cela sera fatal aux Français. L'absence répétée de coordination entre la cavalerie, l'infanterie et l'artillerie tout au long des affrontements le sera également.

Les conséquences politiques de la bataille vont expliquer en grande partie l'attrait pour cette dernière. La seconde abdication ${ }^{1}$ de Napoléon, le 21 juin 1815, marque en effet l'effondrement du rêve impérial d'unification européenne par des conquêtes, sous l'égide de la France. Dorénavant, 1'ordre qui se met en place en Europe est celui du Congrès de Vienne, d'abord libéral sous l'influence notable du tsar Alexandre Ier de Russie puis plus répressif à l'égard des idées libérales, avec l'Autrichien Metternich. Il faudra attendre les révolutions de 1830 puis l'instauration progressive des régimes parlementaires pour que triomphe l'esprit de liberté et d'égalité répandu des années

1 La première abdication de l'Empereur date du 14 avril 1814, à l'issue de la Campagne de France qui devait marquer la fin des conquêtes napoléoniennes en Europe. 
plus tôt par la Grande Armée et un grand nombre de ses officiers ${ }^{1}$. Très vite, le champ de bataille deviendra un lieu visité par les Grands des États vainqueurs comme par les anciens combattants des deux camps et les badauds.

\section{De la bataille au mythe}

Mais, pour aussi forte qu'elle ait pu être ressentie, la victoire des Maisons Royales sur Napoléon, et sur les idées républicaines dont il fut le continuateur, n'explique pas tout l'engouement pour Waterloo. Plusieurs autres éléments, liés autant à la bataille qu'à la légende napoléonienne, entrent en lice. En règle générale, l'analyse des phases successives d'un combat permet de déceler des moments plutôt favorables ou, au contraire, nettement désavantageux pour l'un ou l'autre des protagonistes. En ce qui concerne la bataille de Waterloo, des correspondances structurelles particulièrement intéressantes peuvent être décelées. Leur agencement est conduit à la manière d'un affrontement sans merci entre des adversaires autant tenaillés l'un que l'autre par l'âpre désir de vaincre. Il prépare inexorablement à une issue fatale mais qui n'est pas annoncée d'entrée de jeu.

Et puis, quelle que soit cette issue, la succession des événements est telle que l'honneur des deux camps s'en tire sauf. Qu'on en juge : à l'exaltation initiale des troupes impériales répond l'attente inquiète des Anglo-alliés; si l'attaque du 1er Corps d'infanterie française échoue lamentablement, la poursuite de celui-ci par la cavalerie lourde britannique se termine par un désastre pour cette dernière ; la cruauté des combats pousse à la fuite des hussards de Cumberland et de plusieurs corps de troupes alliées autant qu'à la débandade finale des troupes françaises; à la fin de la journée, le champ de bataille est dominé par des monceaux de cadavres d'hommes et de chevaux autour des carrés anglais, comme par des masses identiques de mourants autour des carrés de la Vieille garde ; sur le front prussien à Plancenoit, une succession de succès et d'échecs témoignent également de la sauvagerie des combats.

1 Sur ce thème des officiers libéraux, issus de tous les milieux et galvanisés par Napoléon ler, lire un très bel ouvrage qui retrace la destinée de quelques-uns de ces hommes, de la Révolution de 1789 à la bataille de Waterloo: cf. M.-H. LEGRAND, Citoyens Officiers de Napoléon, Le Gras-Fougerais, L'Orée du Bois, 1997. 
Des enjeux politiques majeurs, des correspondances structurelles, l'honneur sauvé des protagonistes, un sacrifice sanglant. Voilà qui est propice à la création d'un mythe. Quelques événements supplémentaires vont donner à celui-ci une force toujours très prégnante aujourd'hui. Une fois disparu de la scène politique et quels que soient les sentiments qu'il ait pu inspirer, l'Empereur en exil va être, peu à peu, idéalisé par divers milieux sociaux. En France même, la répression royaliste (connue dans l'Histoire sous le nom de "terreur blanche") va s'abattre de manière extrêmement virulente sur ses partisans. Plusieurs grognards, dont nombre d'officiers supérieurs, vont fuir jusqu'aux États-Unis d'Amérique, notamment dans la région de Philadelphie. Certains iront jusqu'à tenter d'édifier une cité utopique, Demopolis ("la ville des peuples"), en Alabama. Un peu partout, les soldats revenus à la vie civile vont souvent se muer en chantres d'une aventure qui, bon gré, mal gré, aura aussi été la leur. Et puis viendra le mythe de l'empereur martyr, largement orchestré par Napoléon luimême qui exploita habilement les comportements lamentables de l'administration britannique de Sainte-Hélène à son égard ${ }^{1}$. Avec les Romantiques qui s'emparèrent à leur tour de la légende napoléonienne, la défaite de Waterloo allait être définitivement transformée en victoire posthume.

\section{Du mythe à la mémoire}

Finalement, Wellington, vainqueur du jour avec Blücher, et Napoléon, vainqueur posthume, s'en tirent plutôt bien... Même si, comme le confia un touriste anglais qui ne s'y attendait guère, "il n'y en a que pour l'Empereur à Waterloo!" Or, le fait mérite d'être souligné, aucun de ces célèbres protagonistes n'a été personnellement honoré sur le site par un monument officiel. Pas même le maréchal Ney qui se démena comme un diable pendant les combats, ni Cambronne dont le "mot" passa pourtant à la postérité, ni le général von Gneisenau, qui contribua de manière décisive au succès des Alliés. Les stèles et les plaques commémoratives dispersées sur le champ de bataille évoquent bien nominalement quelques morts parmi les généraux et autres officiers. Mais, la plupart d'entre elles portent plutôt sur les masses anonymes de soldats, artilleurs, cavaliers ou

1 Cf. J. Tulard, Napoléon. Le pouvoir, la nation, la légende, Paris, Librairie générale française, 1997. 
troupiers de tel ou tel régiment, qui enlevèrent une position, s'écrasèrent contre une ligne de défense ennemie ou résistèrent à un assaut.

Les stèles et autres monuments commémoratifs qui émaillent le champ de bataille ont d'abord été érigés par les vainqueurs. Le plus ancien d'entre-eux, le Monument Gordon, fut élevé à proximité de La Haie-Sainte en 1817, à la mémoire d'un colonel aide de camp de Wellington. L'année suivante, des officiers de la King's German Legion érigèrent le Monument aux Hanovriens, en l'honneur de leurs camarades disparus sur le champ de bataille. Le Monument prussien fut construit à Plancenoit en 1819, à l'emplacement d'une batterie d'artillerie française qui causa de sérieux dommages aux troupes de Blücher. Entre 1824 et 1826, Guillaume d'Orange entreprit l'élévation de la Butte du Lion, symbole de la victoire, à l'endroit même où son fils fut blessé au cours des combats. Ce vaste chantier allait être mené aux dépens du célèbre "Chemin creux" dans lequel Victor Hugo vit la cause de l'échec des charges de la cavalerie napoléonienne. Il faudra attendre 1904, et une initiative privée, pour que la mémoire des vaincus soit également honorée : l'Aigle blessé, dédié "Aux derniers combattants de la Grande Armée", fut élevé non loin des bâtiments de la Belle-Alliance, là où fondirent quelques-uns des derniers carrés de la Garde Impériale. Les Belges tués dans les combats du 18 juin n'eurent quant à eux leur propre stèle qu'en 1914.

Car ici, à Waterloo, la grandeur des chefs, l'épopée elle-même et le mythe impérial se fondent dans la destinée collective des anonymes. Ainsi, sans même éprouver une quelconque sympathie pour les chefs qui se sont affrontés, tout visiteur du champ de bataille peut se sentir interpellé par l'aventure des combattants du 18 juin 1815. A fortiori, les patriotes des divers camps et les passionnés, mais aussi tous les gens en quête de héros, de mythes et d'allégories, tous ceux qui ont tenté un jour, en vain, de lutter contre la fatalité ${ }^{\text {. }}$

\section{Une mémoire à facettes multiples}

La bataille de Waterloo a frappé les esprits. Très vite, son évocation a débordé les cabinets diplomatiques et les cénacles militaires

1 Sur ce thème de la fatalité qui pousse à des connivences entre la destinée de Napoléon et les destinées individuelles, cf. : M. AUGÉ, L'impossible voyage. Le tourisme et ses images, Paris, Payot \& Rivages, 1997. 
pour atteindre tant le quotidien des gens que leur imaginaire. Vaisselles et gravures ont représenté diverses phases de la bataillel. Des poètes et des écrivains ont immortalisé les scènes les plus tragiques et les nombreux héros de cette mémorable journée ${ }^{2}$. Plus de quatre-vingt-dix villes, quartiers de villes et villages, dont la plupart se trouvent en Angleterre et aux États-Unis, portent le nom de Waterloo dans le monde. Quelques films ont tenté d'offrir une reconstitution plausible des combats ${ }^{3}$. Aujourd'hui, plusieurs sites belges, français, allemands et anglo-saxons consacrés sur Internet à l'épopée napoléonienne, aux wargames, au maquettisme ou encore à l'uniformologie accordent une large place à cette bataille qui entraîna la chute définitive de Napoléon ${ }^{4}$. Depuis plus d'un siècle et demi, de très nombreux ouvrages sont consacrés au sujet, sans épuiser l'intérêt pour l'événement, ni les questions, ni les mystères qui lui sont liés.

Des gens se documentent toujours sur les différentes facettes de la bataille, jusqu'à la passion. Ils y sont aidés par les nombreuses associations qui cultivent le souvenir des combats, effectuent des recherches sur ceux-ci, éditent des publications scientifiques, proposent des promenades, apposent des plaques commémoratives sur les monuments et en divers points du champs de bataille ${ }^{5}$. D'autres

1 Cf. A. Berkeley \& Fl. MichotTe, Waterloo à travers les porcelaines et les faiences. Catalogue de l'exposition organisée par Les Amis du Musée Wellington et le Waterloo Committee, Waterloo, Musée Wellington, 1998 ; Musée Wellington, Waterloo 1815. Une exceptionnelle collection d'aquarelles et de gravures, Waterloo, Musée Wellington, 1998.

2 Parmi les écrivains, nous mentionnerons tout spécialement Sir Walter Scott, créateur du roman historique, Lord Byron, Stendhal (avec La Chartreuse de Parme) et puis, bien sûr Victor Hugo lui-même (avec Waterloo, morne plaine et Les misérables).

3 Le plus célèbre de ces films est incontestablement Waterloo du russe Serguei Bondartchuk (avec Rod Steiger, Christopher Plummer, Orson Welles, Moscou, 1970).

4 Fondation Napoléon: http://www.napoleon.org/fr/presentation.html Freundeskreis Napoleonische Geschichte : http:/hp.citystar.de/fng/ Hat Industrie: http://www.hat.com/ Histoire et Figurines: http://www.histofig.com/ HomePage JensNage Hougoumont: http://members.aol.com/jensnaje/ Mycroft's Brother: http://www.chez.com/mycroft/ The Napoleon Series: http://www.ping.be/napoleon.series/ Waterloo 1815: http://home.t-online.de/home/a.kopp/ Waterloo1815. Le Centre du Visiteur : http://www.braine-lalleud.com/waterloo/

5 A propos des guides, cf. notamment :

- Association franco-européenne de Waterloo, La promenade impériale. Itinéraire commenté des plaques et des stèles hérigées par l'Association franco-européenne de Waterloo avec le concours de la Fondation Napoléon, Louvain-la-Neuve, 1998. 
personnes effectuent de minutieuses recherches -parfois hors de toute motivation académique et muséale et, en tous cas, absentes de buts lucratifs- afin de recréer les diverses phases des affrontements entre les belligérants. Des maquettes aussi fidèles que possible existent dans l'intimité d'une chambre ou d'un grenier comme dans certains musées. L'une d'entre elles est montrée à Londres, au National Army Museum, et met en scène plus de 70.000 figurines illustrant l'échec de l'assaut de la Garde impériale sur les lignes anglaises. Un autre diorama gigantesque existe au Musée de la Figurine Historique de Compiègne. En Allemagne même, le château de Sommershenburg vient d'abriter une exposition centrée sur deux maquettes qui mettent en scène près de 30.000 figurines peintes à la main par deux férus d'histoire napoléonienne.

Assurément, les motivations qui poussent les gens à s'intéresser à la Bataille de Waterloo et à son site doivent être extrêmement diverses. L'événement qui fut à l'origine de l'engouement pour l'endroit est guerrier, fruit de la confrontation idéologique et militaire entre plusieurs États et peuples européens. Aussi ne doit-on pas s'étonner de retrouver parmi les visiteurs des spécialistes ès-stratégie, des gens qui soient motivés par l'amour de l'histoire ou passionnés par l'épopée napoléonienne. Pour beaucoup d'entre eux, le champ de bataille est un vaste cimetière qui impose le respect et qui inspire autant l'exaltation de la mémoire nationale que le resserrement des communautés autour de ceux qui se sont un jour sacrifiés pour elles ${ }^{1}$. Il est même possible que certains parmi ces derniers soient, aujourd'hui encore, parcourus de considérations militaristes et revanchardes.

Néanmoins, le Lion et les alentours drainent aussi -et peut-être surtout- nombre de touristes et de promeneurs dominicaux, qui

- A. Calbert, M. Gérard, J. Mevisse, J.-P. Stiernet, P. Desenfants, Promenade 1815. Circuit pédestre et cycliste sur le Champ de Bataille à Braine-l'Alleud, Genappe, Lasne et Waterloo, Bruxelles, Fédération Touristique du Brabant, 1981.

- J. LOGIE, P. LOZE \& Y. VANDER CRUYSEn,Waterloo. La bataille et les musées. Promenade dans le champ de bataille. Les communes de Braine-l'Alleud, Genappe, Lasne, Waterloo, Tournai, Casterman, 1995.

- Musée Wellington, Musée de Waterloo \& J.-M. Liesse, Musée Wellington. Guide du musée, Waterloo, Les Amis du Musée Wellington/JML Marketing \& Communication, 1998.

- G. SPEECKAERT, P. \& I. BAECKER, Les 135 vestiges et monuments commémoratifs des combats de 1815 en Belgique. Inventaire-Description-Textes-74 illustrations-4 cartes, Waterloo, Waterloo-Relais de l'Histoire asbl, 1990.

1 Cf. L.-V. Thomas, Rites de mort. Pour la paix des vivants, Paris, Fayard, 1985. 
n'entretiennent qu'un rapport assez lointain avec tout cela. Situé aux abords de la capitale et offrant un cadre champêtre assez bien préservé, le site attire en effet des familles entières comme des promeneurs solitaires, des vacanciers embarqués dans un trip d'une demi-journée par divers tour-operators, comme des hommes d'affaires venus y chercher un moment de détente. Les intérêts des uns et des autres ne se rejoignent pas forcément. Que dire alors des quelques soixante-huit nationalités qui se côtoyent aux alentours du Lion ? Un bref parcours des publications consacrées à la bataille, la consultation des livres d'or mis à la disposition du public à l'entrée de divers musées, l'écoute flottante des visiteurs et de leurs guides, quelques échanges avec l'un ou l'autre commerçant du site suffisent pour établir la diversité des motivations.

Les Belges, dont les ancêtres (toutes communautés linguistiques confondues, contrairement aux idées reçues) se retrouvèrent dans les deux camps, ne vibrent apparemment pas tous aujourd'hui du même intérêt pour la bataille. A ne s'en tenir qu'aux ouvrages parus sur les événements de 1815 en Belgique et aux dossiers proposés dans la presse sur les diverses commémorations auxquelles ceux-ci ont donné lieu, les flamands seraient moins intéressés par Waterloo que les francophones ${ }^{1}$. Il est vrai qu'un grand nombre parmi ces derniers vivent sur le théâtre même du passage des troupes et des combats. L'imaginaire wallon serait-il pour autant plus nourri par l'épopée des grognards que celui d'autres peuples? Les marches folkloriques de l'Entre-Sambre-et-Meuse, dont les racines plongent dans les processions du Moyen-Age, font en tout cas une très large place aux uniformes napoléoniens. Chaque année, certains de ces marcheurs organisent en juin une marche du souvenir qui les mène de Jumet, dans la banlieue nord de Charleroi, à Mont-Saint-Jean, sur les pas des troupes impériales. Le Lion lui-même a cristallisé plus d'une fois l'attention des Belges. Symbole du pouvoir néerlandais, il fut vilipendé par les indépendantistes de 1830 . A leur tour, les nationalistes flamingants de la fin du $\mathrm{XIX}^{\mathrm{e}}$ siècle le prirent comme symbole de l'éclatante victoire des races germaniques sur les Latins et les autres. Plus près de nous, il fut voué à la destruction ou à être retourné contre le Nord par quelques nationalistes wallons. Aujourd'hui, des

1 Cf. L. DE Vos, Les 4 jours de Waterloo. 15-16-17-18 juin 1815, Braine-l'Alleud, J.M. Collet, 1997. 
hommes politiques locaux le présentent comme un symbole de l'unité européenne.

Parmi les étrangers qui visitent le site, les Anglais et les Français sont bien évidemment les plus nombreux. Mais si les premiers viennent plutôt célébrer une victoire et leur vainqueur, les seconds en sont sans doute encore à contempler amèrement le lieu où, sans compter sur un malheureux concours de circonstances, ils auraient dû gagner. Quoi qu'il en soit, tous peuvent sans honte aucune, trouver aujourd'hui dans les stèles et monuments qui parsèment le site, le rappel tangible du sacrifice sanglant et de l'hérö̈sme sans faille de leurs ancêtres. La fibre patriotique des uns et des autres n'en est donc pas flétrie. Quant aux Allemands, la discrétion qu'ils affichent dans cette affaire est surprenante. Comme si la victoire, à laquelle Blücher et ses troupes contribuèrent de manière décisive, ne les concernait guère. L'Histoire récente leur aurait-elle appris à mesurer les effets pervers du patriotisme ? Sans doute. Mais il faut noter que l'on compte parmi eux, comme parmi les Russes et même les Anglais, des admirateurs inconditionnels de l'épopée impériale qui participent du côté français aux grandes reconstitutions quinquennales sur le site de la bataille.

Parmi les autres Européens qui visitent le site, les Polonais sont en quête de tout ce qui peut les rattacher à l'Occident, eux les alliés inconditionnels de l'Empereur des Français en 1815 (mais aussi des Anglais quelques guerres plus tard). Les Américains sont également en quête de racines et visitent tout. Les Japonais suivent, appareilsphotos en bandoulière, ne sachant pas trop si les événements commémorés ici se déroulèrent au cours de la Première Guerre Mondiale et si Napoléon était dans le camp des Anglais. En dehors de cet anachronisme, la plupart des visiteurs n'ont qu'une connaissance évasive des événements qui se sont déroulés entre Mont-Saint-Jean, Plancenoit et La Belle-Alliance. L'interprétation des faits et leur impact sur la politique des États européens échappe de même au plus grand nombre.

Certes, plusieurs petits guides, des panneaux didactiques et les musées eux-mêmes apportent l'information nécessaire à la compréhension du site (encore que les musées soient plutôt fréquentés par les passionnés et une forte minorité de personnes instruites ou cultivées). Malgré cet effort pédagogique et les divers monuments qui sollicitent la mémoire des gens et campent tant bien que mal l'ambiance d'une épopée guerrière, l'interprétation de la bataille qui s'est déroulée sur 
ces terres reste très aléatoire. Ne peut-on, en effet, être surpris par toutes ces opinions, glanées au hasard des promenades, qui réduisent les combats à un affrontement entre Bonaparte et Wellington, entre le Mal (quand l'amalgame n'est pas fait entre l'Empereur et Hitler luimême) et le Bien, entre un militariste et un partisan de la paix, entre un despote et un défenseur de la liberté ? Le manichéisme se porte bien et certaines analogies sont, pour le moins, audacieuses. Sans doute faut-il quand même ajouter que, parmi les visiteurs, Napoléon a surtout ses partisans, probablement plus nombreux que les détracteurs. Quoi qu'il en soit, s'il peut paraître périlleux de faire la part des opinions favorables ou défavorables à l'égard de l'un ou l'autre des protagonistes, force est de constater qu'à Waterloo, Napoléon est, de toute façon, mieux connu et surtout plus présent que Wellington. Par un singulier renversement du cours de l'Histoire, c'est la figure de l'Empereur déchu qui domine et, derrière elle, le mythe napoléonien lui-même.

\section{Et du mythe au rituel}

Le visiteur qui se rend sur le site du champ de bataille accède-t-il pour autant, in situ et de visu, à cette dimension épique et symbolique qui dépasse l'historiographie? Assurément, nous n'en sommes plus au lendemain de la bataille, ni dans les mois qui la suivirent. A cette époque-là, on pouvait encore presque tout voir, des cadavres, des blessés, les destructions. L'odeur pestilentielle qui régna longtemps sur la "morne plaine" rappelait aux passants l'horrible atmosphère des combats qui venaient de s'y dérouler. Il était dès lors forcément difficile de rester insensible au spectacle de la souffrance et de la misère humaine. Mais, près de deux siècles plus tard, on n'en est évidemment plus là. Le site a-t-il pour autant perdu de son pouvoir évocateur ; s'est-il transformé en un gigantesque parc d'attraction ou réduit à un musée de personnages momifiés ?

Les $20 \mathrm{~km}^{2}$ du champ de bataille restent, dans l'ensemble, particulièrement bien conservés. Les grosses fermes d'Hougoumont, de la Haie-Sainte et de la Papelotte, anciens bastions avancés des forces britanniques et de leurs alliés, se dressent toujours dans leur environnement champêtre. La chaussée reliant Charleroi à Bruxelles découpe encore les lieux en deux espaces caractéristiques. A droite, vers Ohain et Wavre, le site qui fut occupé, avec des bonheurs divers 
et jusqu'à l'arrivée des Prussiens de Zieten, par les troupes du Ier Corps de Drouet d'Erlon, offre un relief toujours assez tourmenté. A gauche, vers Braine-l'Alleud, les champs qui subirent les grandes charges de cavalerie puis l'assaut infructueux de la Garde, révèlent encore la douceur ondulée de leurs pentes. Ici et là subsistent quelques chemins de terre et, moins nombreux qu'alors, l'un ou l'autre bosquet.

Néanmoins, et bien que le paysage ne puisse plus être modifié sur près de 500 hectares depuis 1914 , plusieurs endroits du site ont dû céder aux pressions de la modernité. Le village de Plancenoit qui fut, dans la soirée du 18 juin, le théâtre d'un épouvantable carnage, s'est donné aux lotissements résidentiels. Seuls l'église et le cimetière gardent encore l'austère aspect d'un mausolée. Dancing et club privé jouxtent l'Aigle blessé à La Belle-Alliance, alors que la chaussée qui longe le monument a dû être élargie pour canaliser un trafic routier de plus en plus dense. Quelques maisons ont été bâties aux abords de la même voie, mais un peu plus au nord, dans les Fonds de la HaieSainte. Les bâtiments massifs d'un couvent se dressent depuis 1929 , tout près de la Papelotte, sur l'ancienne ligne de défense britannique. Le quartier de Mont-Saint-Jean est traversé par une autoroute et l'ancienne commanderie de Malte, qui fut l'hôpital des Anglais pendant la bataille, est bordée par une grande surface commerciale. La Butte du Lion, visible par beau temps à plus de $20 \mathrm{~km}$ aux alentours, regroupe à ses pieds le Centre du Visiteur, le Musée du Panorama, le Musée de cire, une plaine de jeux, des boutiques à souvenirs, trois grands parkings, quelques cafés et restaurants.

Bien des critiques ont dénoncé la difficulté de l'accès à l'espace du champ de bataille. Et de fait, pour qui vient de Bruxelles, de Braine-l'Alleud ou du cœur urbain de Waterloo, un dédale de sorties autoroutières, d'artères commerciales, de voies rapides, de carrefours et de ronds-points animés par une incessante circulation automobile, s'offre à lui. Le touriste qui arrive de Nivelles ou de Mons aura presque autant de difficulté à se dégager d'une autoroute encaissée ou d'un oppressant tissu urbain, tout au moins à l'approche du site proprement dit. Celui qui vient de Charleroi, par contre, sera plus gâté que les précédents car il aura eu la possibilité de découvrir un bref instant l'étendue qui mène à l'ancienne ligne de défense anglo-alliée. Mais, canalisé par une chaussée où le trafic est également très dense, il n'aura probablement pas pu deviner qu'il circule effectivement sur le champ de bataille depuis un bon moment déjà. Il aura laissé sur sa droite l'ancienne ferme du Caillou, dernier quartier général de 
Napoléon, aujourd'hui transformée en musée. Il sera passé sans même le savoir devant le monticule qui servit d'observatoire à l'Empereur et d'où il aurait pu découvrir une vue superbe sur le front anglais (si, tout au moins, l'endroit avait été débroussaillé et mis en valeur). Arrivé à la hauteur de la Colonne commémorant le passage de Victor Hugo, il prendra sans doute conscience qu'il est enfin sur les lieux des combats. Mais, pris en tenaille par un poids lourd et une file de voitures, il lui sera pratiquement impossible de s'arrêter pour rendre hommage à l'Aigle blessé.

Force est de constater que seule la Butte du Lion et le hameau qui l'entoure jouissent d'une visibilité et d'une identification claire. C'est donc forcément là que se retrouvent l'ensemble des visiteurs et des pèlerins. C'est éventuellement à partir de là que les plus téméraires, les mieux renseignés, tous ceux qui ont du temps, partiront à la découverte des autres monuments du site. Mais il est peu probable qu'ils se déplacent tous au-delà du carrefour, à proximité de la HaieSainte, ou qu'ils empruntent l'ancien chemin creux, qui mène à Hougoumont. La plupart des gens feront l'impasse sur Plancenoit, la Papelotte et le hameau de Smohain. Très peu d'entre eux tenteront la visite des musées périphériques qui regorgent pourtant de souvenirs. La focalisation sur le Lion et les alentours immédiats est un avatar de l'histoire et consacre d'une certaine façon le triomphe d'une masse -la Butte- sur l'environnement. Certes, l'endroit a été le théâtre de plusieurs épisodes tragiques de la bataille. Mais d'autres lieux, ailleurs sur le champ de bataille, l'ont été tout autant, voire davantage. La résistance des Anglais à Hougoumont, celle des Français dans le cimetière et l'église de Plancenoit, ou aux abords de la Belle-Alliance, mériteraient un aménagement tout aussi digne que celui dont a bénéficié l'endroit où un prince fut blessé au bras.

La découverte des moindres recoins du théâtre des combats restera une aventure individuelle réservée aux spécialistes et aux passionnés. Pour tous ceux qui sont empreints de références, de lectures et d'images fortes, le mythe est accessible partout et le respect envers ceux qui reposent à leurs pieds dicte la conduite.

Mais, pour tous les autres, la plongée dans l'histoire et l'éventuelle rencontre avec les héros du drame, parfois jusqu'à la fascination, s'opère ailleurs, là où s'impose la masse : à la Butte du Lion et dans les alentours transformés en mémorial. Pour eux, tout se passe comme si Napoléon, Wellington, Blücher et leurs troupes, inapprochables ailleurs, s'étaient symboliquement retrouvés en cet endroit. 
Au point que l'on ne sache plus très bien aujourd'hui qui d'entre eux -sinon tous- est commémoré par l'imposant monument. Du reste, la proximité de nombreuses stèles, les enseignes des restaurants et des tavernes, les magasins de souvenirs dissipent tout malentendu: l'endroit est napoléonien et évoque d'anciens exploits guerriers.

\section{Du regard à la postérité}

D'un premier parcours visuel, le visiteur conservera sans doute l'image de maisons aux façades blanches, d'un mannequin de grognard, de bibelots et autres figurines militaires aux vitrines des magasins, de quelques parkings ombragés. Peut-être aura-t-il aussi distraitement mis en mémoire telle ou telle inscription au hasard des monuments rencontrés. Une visite rapide du Musée de cire lui aura probablement permis de se familiariser davantage avec les silhouettes des principaux protagonistes. La fresque panoramique de la rotonde, au pied du Lion, ne le laissera certainement pas insensible. Car là, vraiment, plongé dans l'atmosphère de la bataille, submergé par les charges des lanciers et des cuirassiers qui viennent mourir à ses pieds, il touchera à l'épopée. Sans doute s'attardera-t-il ensuite à la librairie du Centre du Visiteur avant de replonger dans les tirs de mitraille que lui propose un spectacle audio-visuel. Enfin, gorgé de couleurs et de sons, un peu plus au fait des événements qui secouèrent les lieux et de leur chronologie, ressentant confusément en lui une étrange anxiété à l'égard de la fatalité, il va lui aussi entreprendre l'ascension de la Butte du Lion.

Dans cette découverte déambulatoire, qui est autant une promenade distrayante qu'un processus singulier de connaissance, l'apprentissage de l'histoire -la rencontre du mythe- passe aussi par un effort physique. Or, l'accès au Lion, principale attraction du site, est d'abord une petite prouesse sportive au terme de laquelle le mythe lui-même est à portée. C'est après avoir gravi 226 marches, qu'en effet, le visiteur atteindra pleinement l'ensemble du champ de bataille. Le savoir acquis en contrebas ou, auparavant, en d'autres lieux, va s'ouvrir alors à une autre dimension : celle du regard et des sensations physiques. Se perdant un bref instant dans la contemplation des étendues, le visiteur va devenir stratège, dominer ce qui fut la fournaise, entrer dans le mythe et, sans doute aussi, s'il y est disposé, donner libre cours à son rêve. C'est alors que se mêleront les éléments 
d'histoire qu'il aura retenus, l'épopée des héros et la destinée individuelle.

\section{Considérations finales}

Chacun à sa manière pourra donc retrouver à Waterloo ce qu'il cherche, ce qu'il croit, ce qu'il est prêt à vivre et éventuellement à partager avec tout ceux qui l'accompagnent. Ici, personne n'est véritablement emprisonné par l'univocité d'un message. C'est ce qui fait la force du site et, incontestablement la clé de son succès. Mais, qu'on le veuille ou non, Waterloo reste d'abord et avant tout un grand moment d'Histoire. Dans ce domaine, la puissance évocatrice du site, tout rétréci qu'il soit à la Butte du Lion et à ses alentours, ne peut être sous-estimée. Or, s'il appartient aux contemporains eux-mêmes d'entretenir la flamme du souvenir des événements qui se sont déroulés entre Mont-Saint-Jean, Hougoumont et Plancenoit (et l'on sait que bien des améliorations pourraient être apportées aux infrastructures d'accueil actuelles) il serait vain de fixer l'Histoire. Aujourd'hui, et bien que toutes les blessures du passé ne soient pas encore totalement cicatrisées, les anciens belligérants président ensemble, après bien d'autres aventures communes, aux destinées de l'Union européenne. Un autre sens, dégagé de ce nouveau combat, peut être donné au sacrifice des grands ancêtres. Ainsi triomphe la Vie. Le mythe de l'Aigle ne s'effilochera pas pour autant... ni d'ailleurs l'écho que lui donneront encore maintes destinées individuelles. Et le Lion luimême, qui d'ailleurs ne se dresse plus contre la France depuis bien longtemps, pourra de longues années encore drainer rêves et regards de la morne plaine. 